BMJ Open

Diabetes

Research

\& Care

\title{
Internet-delivered cognitive behaviour therapy for depression in people with diabetes: study protocol for a randomised controlled trial
}

\author{
Lisa Robins, ${ }^{1,2,3}$ Jill Newby, ${ }^{2,4}$ Kay Wilhelm, ${ }^{1,2,3}$ Jessica Smith, ${ }^{4}$ Therese Fletcher, ${ }^{1}$ \\ Trevor Ma, ${ }^{3}$ Adam Finch, ${ }^{3}$ Lesley Campbell, ${ }^{5}$ Gavin Andrews ${ }^{2,4}$
}

To cite: Robins L, Newby J, Wilhelm K, et al. Internetdelivered cognitive behaviour therapy for depression in people with diabetes: study protocol for a randomised controlled trial. BMJ Open Diabetes Research and Care 2015;3: 0000144

doi:10.1136/bmjdrc-2015000144

Received 30 July 2015 Revised 9 October 2015 Accepted 20 October 2015

\section{(a) CrossMark}

${ }^{1}$ Faces in the Street, St Vincent's Health Australia, Sydney, New South Wales, Australia

${ }^{2}$ Faculty of Medicine, School of Psychiatry, University of New South Wales (UNSW), Darlinghurst, New South Wales, Australia

${ }^{3}$ Consultation Liaison Psychiatry, St Vincent's Health Australia, Darlinghurst, New South Wales, Australia ${ }^{4}$ Clinical Research Unit for Anxiety and Depression (CRUfAD), St Vincent's Health Australia, Darlinghurst, New South Wales, Australia ${ }^{5}$ Diabetes Centre, Garvan Institute, St Vincent's Health Australia, Darlinghurst, New South Wales, Australia

Correspondence to Dr Jill Newby; j.newby@unsw.edu.au

\section{ABSTRACT}

Introduction: Depression substantially contributes to the personal burden and healthcare costs of living with diabetes mellitus (DM). Comorbid depression and DM are associated with poorer quality of life, poorer selfmanagement and glycemic control, increased risk for DM complications and higher mortality rates, and higher health service utilization. Depression remains underrecognized and undertreated in people with DM, which may, in part, result from barriers associated with accessing face-to-face treatment. This study will examine the efficacy of an internet-based cognitive behaviour therapy programme for major depressive disorder (iCBTMDD) in people with DM.

Methods and analysis: A CONSORT 2010 compliant, registered randomised controlled trial of the intervention (iCBT-MDD) versus a treatment as usual control group will be conducted. The study will include 100 adults aged 18 years and over with a diagnosis of type 1 or type 2 DM and self-reported symptoms that satisfy MDD which will enable us to detect a statistically significant difference with a group effect size of 0.6 at a power of $80 \%$ and significance level of $p=0.05$. Participants will be randomised to receive the iCBT-MDD programme immediately, or to wait 10 weeks before accessing the programme. Primary outcomes will be self-reported depression severity, DM-related distress, and glycemic control (glycosylated hemoglobin). Secondary outcomes will be general distress and disability, generalized anxiety, lifestyle behaviours, somatization, eating habits, alcohol use, and acceptability of the iCBT programme to participants, and practicality for clinicians. Data will be analyzed with linear mixed models for each outcome measure.

Ethics and dissemination: The Human Research Ethics Committee of St Vincent's Hospital Australia have given ethics approval (HREC/13/SVH/291). Results will be disseminated via peer-reviewed publication and social media channels of Australian Diabetes Consumer Representative Bodies.

Trial registration number: The trial is registered with the Australian and New Zealand Clinical Trials Registry (ACTRN12613001198718).

\section{BACKGROUND}

The international burden of diabetes mellitus (DM) is significant in personal as well as

\section{Key messages}

- This randomised controlled trial evaluates the impact of a generic online cognitive behaviour therapy (CBT) programme for depression in people with diabetes.

- We will examine the efficacy of the programme, moderators of treatment response, and acceptability to patients.

- This study will answer the question of whether generic online CBT programme for depression are effective and acceptable to people with comorbid diabetes and depression.

financial terms, accounting for 5.1 million deaths and $11 \%$ of worldwide health spending in 2013. ${ }^{1}$ Depression makes a substantial and independent contribution to the personal burden of $\mathrm{DM}^{2}{ }^{2}$ Compared with people with DM without depression, those with comorbid diabetes and depression have poorer quality of life, ${ }^{3}{ }^{4}$ higher DM-related distress, ${ }^{5}$ and poorer self-management of their condition. ${ }^{6}$ Depression in diabetes is also associated with increased healthcare utilization $^{7} 8$ and expenditure, ${ }^{2} 9$ and poorer medical outcomes, including worse glycemic control, ${ }^{10}{ }^{11}$ increased risk for DM-related complications, and higher mortality rates. ${ }^{12} 13$

\section{Depression treatment in DIM}

A recent Cochrane review and meta-analysis of treatments for depression in people with DM showed that pharmacological and psychological interventions are effective ${ }^{14}$ with moderate and clinically significant effects on depression symptoms. Of the available psychological treatments, cognitive behaviour therapy (CBT) has received the most support. Meta-analyses indicate that psychological interventions including CBT have a positive effect in reducing depression symptoms and remission rates, but may also be 
effective at reducing glycosylated hemoglobin (HbAlc) in the long term. ${ }^{15}$ These results suggest that treatment for depression not only improves quality of life, but can have a positive effect on DM management.

Although effective treatments are available, depression is under-recognized and undertreated in people with DM. $^{2}{ }^{16}$ Recent evidence has suggested that a lack of recognition of depression symptoms by practitioners may in part underpin this shortfall in treatment. ${ }^{17}{ }^{18}$ Financial barriers have been cited as another factor limiting access to care for a substantial minority of people living with DM. ${ }^{19}{ }^{20}$ In addition, appropriate treatment options must be accessible following screening for depression in diabetes: ${ }^{21}$ an important consideration given access to face-to-face mental health services is restricted or unavailable for some. Internet-based programmes have the potential to overcome these barriers in delivery of mental health services in routine DM care.

\section{Online CBT for depression in DM}

Online CBT for depressive symptoms appears to be as effective as face-to-face delivery ${ }^{22}$ at a fraction of the cost and clinical time. ${ }^{23}$ When implemented as part of routine clinical care, outcomes for internet-delivered CBT (iCBT) for depressive symptoms are equivalent to those observed in the controlled trials. ${ }^{24}{ }^{25}$ Importantly, there is also evidence to suggest that iCBT for depression is acceptable to participants, regardless of age, and reduces the disability associated with this serious mental illness, increases workforce participation, and reduces suicide risk. ${ }^{26}$

It is currently unclear whether people with comorbid depression and DM are best served by a general online depression programme or a depression programme with content tailored to people with diabetes. It has been argued that a general programme may not sufficiently address the disease-specific emotional distress of living with DM, which appears to be an overlapping, yet distinct construct from depression or general distress. ${ }^{27}$ DM-related distress, which concerns negative emotional reactions related to living with $\mathrm{DM}$, is far more common and chronic than depression, and is more closely associated with DM self-care and HbAlc. ${ }^{27-30}$ As DM-related distress seems to mediate the association between depression and glycemic control, ${ }^{31}{ }^{32}$ it is thought that tailored interventions may be more effective in improving both psychological and medical outcomes. ${ }^{33}$

Two randomised controlled trials (RCTs) have examined guided online CBT for depression, both of which were tailored to people with DM. The first trial of 255 Dutch adults with type 1 (T1) and type 2 (T2) DM, found that an online guided CBT for depression programme with DM-specific content was more effective than treatment as usual (TAU) in reducing depression symptoms, increasing depression remission rates, and reducing DM-related distress, with small-to-moderate effect sizes. ${ }^{34}$ There were no significant differences in glycemic control between groups 2 months post-treatment completion. ${ }^{34}$ Secondary analyses found that MDD, anxiety disorder, and elevated DM distress were not significant effect modifiers, suggesting the guided CBT for depression programme was suitable for participants with clinical profiles ranging from subclinical to severe mental health problems. ${ }^{35}$ The second trial with 260 German adults with T1 and T2 DM found that a guided web-based intervention to reduce depression with DM-specific content was effective in reducing both depressive symptoms and DM-specific emotional distress when compared to a brief online unguided psychoeducation programme for depression. ${ }^{36}$ Changes in glycemic control were not measured.

These trials show promising results for treating psychological symptoms in people with DM with online CBT for depression programmes, but provide no evidence that additional tailoring with DM-specific content improves glycemic control. The efficacy of an unmodified, general online depression programme has not yet been tested in people with DM. Such work is required to ascertain whether an untailored, general online depression programme is efficacious and acceptable in a population of people with DM, and to help substantiate whether additional intervention tailoring is indeed warranted if outcomes are not equivalent. This research will also identify responders and non-responders to unmodified online CBT depression programmes, which will assist with refining the characteristics of the subgroup of people with DM who may benefit from more targeted treatment approaches.

\section{The current study}

This paper presents the SPIRIT guideline compliant ${ }^{37}$ study protocol for the RCT of the iCBT programme for MDD (iCBT-MDD) in people living with DM, a programme which aims to improve the emotional well-being of people living with depression. The iCBT-MDD programme used in this study has been demonstrated to be efficacious in the general population. ${ }^{38}$ This study replicates the RCT of van Bastelaar et al $2011^{34}$ in an independent sample, and will extend on previous research by examining whether a general online CBT programme for depression without DM-specific content is efficacious for people with DM.

\section{Study objective}

The aim of this RCT will be to evaluate the efficacy of the iCBT-MDD programme for Australian participants with comorbid depression and either T1 or T2 DM. This will enable us to test the generalisability of the results found by van Bastelaar and colleagues to an Australian sample of depressed DM participants. We also aim to identify moderators of treatment effect, including initial severity of depression and presence of DM-related distress, and the differential impact of the programme on depression versus DM-related distress. 


\section{Primary outcomes}

Primary outcomes will be the impact of the iCBT-MDD programme on self-reported depression symptoms, as well as DM distress and self-reported glycemic control (HbAlc levels). Our primary hypothesis is that adults with $\mathrm{T} 1$ and $\mathrm{T} 2 \mathrm{DM}$ randomised to receive immediate treatment will demonstrate significantly lower levels of depression severity than those receiving TAU from preintervention to postintervention. We also hypothesise that relative to TAU, the immediate treatment group will demonstrate: (1) lower levels of DM distress; and (2) better glycemic control.

\section{Secondary outcomes}

Secondary outcomes will be general distress and disability, generalised anxiety, lifestyle behaviours, somatisation, disordered eating, alcohol use, and acceptability of the iCBT programme to participants with DM and depression. Our secondary hypotheses are that compared with those in TAU, the immediate treatment group will demonstrate significant: (1) reductions in general distress, disability, generalised anxiety, somatic symptom severity, and alcohol use; (2) improvement in lifestyle and eating habits. We also hypothesise that the treatments will be acceptable to people with comorbid DM and depression, and practical for clinicians. Recruitment, retention, and intervention completion rates will also be examined.

\section{METHODS/DESIGN}

Design

A CONSORT 2010 compliant, ${ }^{39}$ registered RCT of the intervention (iCBT) versus a TAU control group. This parallel group, two-arm, superiority trial with 1:1 allocation ratio will demonstrate whether the benefit from the iCBT intervention is superior to natural remission and placebo response. Outcomes will be assessed at three time points: baseline, postintervention/wait time and 3-month follow-up (for the iCBT group only). We will also assess depression, general psychological distress and DM distress at the mid time point.

\section{Setting and recruitment}

Participants will be recruited nationwide in Australia via online advertisements posted on national DM-related websites and social media platforms, Facebook, mailing lists, fliers in medical settings, and invitation in the DM Services of St Vincent's Hospital Sydney. Participants apply for the study via the Virtual Clinic website (http://www.virtualclinic.org.au), where they will complete an automated screening questionnaire. Excluded applicants immediately receive an onscreen message and email thanking them for their application, and encouraging them to discuss their symptoms with their physician.

Applicants whose responses meet selection criteria are sent an information sheet via email explaining the study, and invited to participate in a brief phone interview which confirms whether they meet Diagnostic and Statistical Manual of Mental Disorders, Fourth Edition (DSM-IV) criteria for depression using the Mini International Neuropsychiatric Interview V.5.0.0 (MINI). ${ }^{40}$ Excluded participants are sent an email confirming exclusion with information about depression treatment and a suggestion that they discuss their symptoms with their physician. An offer of treatment is made to participants meeting study inclusion criteria, and they are asked to return an electronic consent form to the investigators prior to study enrolment.

\section{Eligibility criteria}

For adults residing in Australia who are aged 18 years and over, eligibility criteria include: a self-reported diagnosis of T1 or T2 DM, access to the internet and a printer, self-identified depression, Patient Health Questionnaire-9 (PHQ-9) scores greater than 5 and less than 24 and meets criteria for MDD according to diagnostic interview, willingness to provide personal and GP contact details, and written informed consent.

Because the intervention and research questionnaires are only available in English language, participants who do not have fluent English skills will be excluded. Participants will also be excluded if they have started CBT in the past month, changed antidepressant medication in the past 2 months, are planning an extended absence during the intervention period, are using atypical antipsychotics or benzodiazepines, or have a selfidentified psychotic disorder or bipolar disorder or substance dependence. Participants scoring 2 or 3 on question 9 of the PHQ-9 and identified as being at significant risk of suicide or deliberate self-harm in a risk assessment conducted by clinicians will also be excluded.

\section{Sample size calculations}

The sample size was calculated on the basis of the primary hypothesis and informed by previous studies comparing iCBT for depression with no-treatment control groups. ${ }^{38}$ With a sample size of 100 participants, the study is powered (at 0.8 power) to detect a group difference of 0.6 on the primary MDD measure at $\mathrm{p}<0.05$. To detect a medium effect size of $0.6,44$ participants are needed in each study condition; recruiting up to 100 participants will account for expected attrition.

\section{Study duration}

Thirty months from September 2013 inclusive of recruitment, follow-up, analysis and write up of results. The primary end point will be the end of active treatment for treatment group 1 (ie, 11 weeks after beginning the programme) and the secondary end point will be the 3-month follow-up of these participants (3 months after completion of active treatment for the iCBT group only; the TAU group will be offered the iCBT programme after 10 weeks). 


\section{Study arms}

Participants will be randomised to one of two treatment arms: iCBT intervention or TAU control. Participants from both treatment arms will continue to receive usual care from their health services. Participants from the intervention arm will receive immediate access to the iCBT-MDD programme. Following randomization, participants in the TAU control condition will have a waiting period of 10 weeks after which they will be given access to the iCBT programme. During the waiting period, they will receive email or phone reminders to complete their questionnaires, but will have no other contact with the research and clinical team until they start the intervention.

\section{Randomization}

Randomization to the two groups will be generated by http://www.random.org and each choice will be placed in an opaque sealed envelope by a researcher who is independent from the study. Allocation will be concealed to the clinician and participant until after selection criteria are met on the phone interview, and the offer of treatment is made and accepted by the participant.

\section{Participant withdrawal}

Participants may be withdrawn from the study if they start or change medication, or if a clinician identifies that the participant requires alternate support in a risk assessment. This is a rare occurrence and will only occur after discussion with the participant. Participants who are withdrawn will not be included in the final analysis.

\section{iCBT-MDD intervention}

The iCBT-MDD programme consists of six fully automated, online lessons involving CBT components such as psychoeducation, behaviour activation, cognitive restructuring, problem solving, graded exposure, relapse prevention, and assertiveness skills. See table 1 for an overview of the content of the programme.

At the end of each lesson, participants are required to download a lesson, summary or 'homework' document. This document summarises the key information in each lesson, as well as tasks that reinforce the content of the lesson. Lessons are completed sequentially. Participants are required to wait a minimum of 5 days between completing a lesson and starting the next lesson. Each participant has 10 weeks to complete the entire six-lesson programme.

The iCBT-MDD programme is clinician-assisted: clinicians are trained clinical psychologists or psychiatrist registrars with either masters or $\mathrm{PhD}$-level qualifications. Clinicians will monitor participant distress levels throughout the intervention, and correspond with participants by email and phone to provide support as required.
Table 1 Internet-based cognitive behaviour therapy for major depressive disorder programme overview

Depression programme content

1 Lesson Psychoeducation about depression, medications, and identifying symptoms of depression

$\begin{array}{lll}\text { HWK } & \text { Identifying symptoms of depression } \\ & \text { Lesson } & \text { Psychoeducation about low activity }\end{array}$ in depression, activity monitoring, thought monitoring (including monitoring rumination), education about cognitive distortions, shifting attention, and sleep

HWK Activity monitoring, thought monitoring

3 Lesson Activity planning, thought challenging/cognitive restructuring; challenging positive and negative metacognitive beliefs about repetitive thinking; hunt for positives

HWK Activity planning, thought challenging, hunt for positives

4 Lesson Structured problem solving, education about avoidance and facing fears

HWK Facing your fears to overcome avoidance

5 Lesson Assertive communication, effective communication skills, hunt for positives, thought challenging

HWK Thought challenging, hunt for positives

6 Lesson Relapse prevention

HWK Relapse prevention plan

General extra resources 100 things to do (ideas for pleasant activity planning), assertiveness, conversation skills, activity planning and monitoring worksheet, facing fears worksheet, positives hunt worksheet, structured problem solving worksheet, thought challenging worksheet, good sleep guide, FAQs (lesson 1-6)

FAQ, frequently asked questions; HWK, homework.

\section{Improving efficacy and adherence}

A recent meta-analysis found that the most effective webbased interventions for depression were those that included some support from a healthcare professional. ${ }^{41}$ Phone and email reminders facilitate adherence and effectiveness in online depression interventions. ${ }^{42}{ }^{43}$ These strategies are incorporated into the iCBT-MDD programme to improve adherence. Specifically, automated emails notify when a new lesson is available, remind participants to complete lessons and to congratulate participants for lesson completion. Clinicians email or call participants who request contact, have a depression or distress score indicating deterioration in their condition, or who have not recently logged into the programme following an automated email reminder. 
Ethical consideration, possible risks and benefits

The Clinical Research Unit for Anxiety and Depression at St Vincent's Hospital has now treated over 1700 patients with this iCBT-MDD programme in efficacy trials, and an additional 1000 patients have participated in effectiveness studies of the same programme. There are no known risks associated with this iCBT programme and clinically significant deterioration is rare. Serious adverse events will be those indicating deterioration in well-being as per questionnaire responses at the time points throughout the programme. Regular team meetings will be conducted to monitor any difficulties participants may be having and ways of best dealing with these difficulties. Serious adverse events will be reported to the Ethics Committee. Possible individual benefits include reduced depressive symptoms, distress, and impairment, and clinically significant remission and recovery from MDD. ${ }^{44}$

\section{Primary outcome measures}

The PHQ- $9^{45}$ is a well-validated self-report depression questionnaire that will be administered online to measure depression severity over the past 2 weeks. The PHQ-9 contains nine items answered on a four-point Likert scale; the total score ranges between 0 and 27 with scores equal or above 10 having a sensitivity of $88 \%$ and a specificity of $88 \%$ for major depression. ${ }^{45}$ Current DSM-IV diagnoses of depression will be assessed with the MINI. ${ }^{46}$ The MINI possesses excellent inter-rater reliability $(\kappa=0.88-1.00)$ and good concurrent validity with the Composite International Diagnostic Interview (CIDI, WHO, 1990). ${ }^{47}$ Glycemic control will be measured via self-reported HbAlc values. While self-reported HbA1c levels may be influenced by biased reporting, we will not have sufficient resources to conduct blood tests to obtain an objective measurement of HbA1c levels. DM-related distress will be measured using the Problem Areas in Diabetes (PAID) ${ }^{48}$ questionnaire, a wellvalidated 20 -item measure with a five-point Likert scale; total scores are multiplied by 1.25 and range from 0 to 100 (with higher scores indicating greater emotional distress). It has demonstrated sensitivity to change ${ }^{49}$ and good internal and test-retest reliability. ${ }^{48}$ See table 2 for an overview of all measurement tools and administration time points.

\section{Secondary outcome measures}

The secondary outcome measures include the: Kessler 10-item Psychological Distress scale (K-10) ${ }^{50}$ for psychological distress; Fantastic Checklist ${ }^{51}$ for lifestyle behaviours, SF-12 $2^{52}$ for disability, functional impairment, and well-being; PHQ-Eating module ${ }^{53}$ for eating habits; PHQ-Alcohol module ${ }^{53}$ for alcohol use disorders; Generalized Anxiety Disorder 7-Item (GAD-7 $)^{54}$ for anxiety severity; and the PHQ-Physical symptoms module $(\mathrm{PHQ}-15)^{53}$ for somatisation symptom severity.

Table 2 Measurement tools and questions at each time point

\begin{tabular}{|c|c|c|c|c|c|}
\hline & Measures & Baseline & Mid & Post & $\begin{array}{l}\text { 3-Month } \\
\text { follow-up }\end{array}$ \\
\hline Demographics & & $\sqrt{ }$ & & & \\
\hline \multicolumn{6}{|l|}{ Process evaluation } \\
\hline $\begin{array}{l}\text { Expectancy of benefit (baseline) and } \\
\text { intervention acceptability and patient } \\
\text { satisfaction (post-treatment) }\end{array}$ & $\mathrm{CEQ}^{38}$ & $\sqrt{ }$ & & $\sqrt{ }$ & \\
\hline \multicolumn{6}{|l|}{ Primary outcomes } \\
\hline Depression & PHQ -945 & $\sqrt{ }$ & $\sqrt{ }$ & $\sqrt{ }$ & $\sqrt{ }$ \\
\hline & $\begin{array}{l}\text { Mini International Neuropsychiatric } \\
\text { Interview V.5.0.0 MDD module }\end{array}$ & $\sqrt{ }$ & & & $\sqrt{ }$ \\
\hline DM distress & PAID $^{48}$ & $\sqrt{ }$ & $\sqrt{ }$ & $\sqrt{ }$ & $\sqrt{ }$ \\
\hline Glycemic control & Self-reported HbA1c & $\sqrt{ }$ & & $\sqrt{ }$ & $\sqrt{ }$ \\
\hline \multicolumn{6}{|l|}{ Secondary outcomes } \\
\hline Psychological distress & $\mathrm{K}-10^{50}$ & $\sqrt{ }$ & $\sqrt{ }$ & $\sqrt{ }$ & $\sqrt{ }$ \\
\hline Lifestyle behaviours & The Fantastic Checklist $^{51}$ & $\sqrt{ }$ & & $\sqrt{ }$ & $\sqrt{ }$ \\
\hline $\begin{array}{l}\text { Disability, functional impairment and } \\
\text { well-being }\end{array}$ & The SF $-12^{52}$ & $\sqrt{ }$ & & $\sqrt{ }$ & $\sqrt{ }$ \\
\hline Eating habits & PHQ-Eating module ${ }^{53}$ & $\sqrt{ }$ & & $\sqrt{ }$ & $\sqrt{ }$ \\
\hline Alcohol & PHQ_Alcohol module ${ }^{53}$ & $\sqrt{ }$ & & $\sqrt{ }$ & $\sqrt{ }$ \\
\hline Anxiety & GAD- $7^{54}$ & $\sqrt{ }$ & & $\sqrt{ }$ & $\sqrt{ }$ \\
\hline Somatization & $\mathrm{PHQ}-15^{53}$ & $\sqrt{ }$ & & $\sqrt{ }$ & $\sqrt{ }$ \\
\hline \multicolumn{6}{|l|}{ Moderators } \\
\hline Attachment style & The Relationships Questionnaire 55 & $\sqrt{ }$ & & & \\
\hline
\end{tabular}




\section{Additional measurements}

We will collect: sociodemographic information (age, gender, education, and occupation); diabetes-related information (type, duration of illness, treatment, and complications) expectancy of benefit (baseline); and intervention acceptability and patient satisfaction (posttreatment) using the Treatment Credibility/Expectancy Questionnaire (CEQ) $;^{38}$ information about selfidentified attachment style using The Relationships Questionnaire; ${ }^{55}$ and data about the method of recruitment (eg, through social media or clinic referrals).

\section{Statistical analysis}

All analyses will be undertaken in SPSS V. 22. Linear mixed models with time as a within-subjects variable and intervention group as the between-subjects variable will be conducted separately for each outcome measure. Within-groups and between-groups effect sizes (Cohen's $\mathrm{d}$ and $95 \%$ CIs around the effect sizes) will be calculated for all outcome measures. For each group, planned contrasts will be used to compare changes from baseline to post-test, and 3-month follow-up.

\section{Trial monitoring and management}

Participants screened out of the study will be encouraged to see their physician and receive an email with depression support information and contact numbers.

Participants in the iCBT-MDD group receive regular email and or phone contact with their clinician until they have completed lesson 2 after which contact will be
Figure 1 CONSORT flow diagram for the randomised controlled trial of the iCBT-MDD programme for people with diabetes.

iCBT-MDD, internet-based cognitive behaviour therapy programme for major depressive disorder; PHQ-9, Patient Health Questionnaire-9-Item; TAU, treatment as usual.

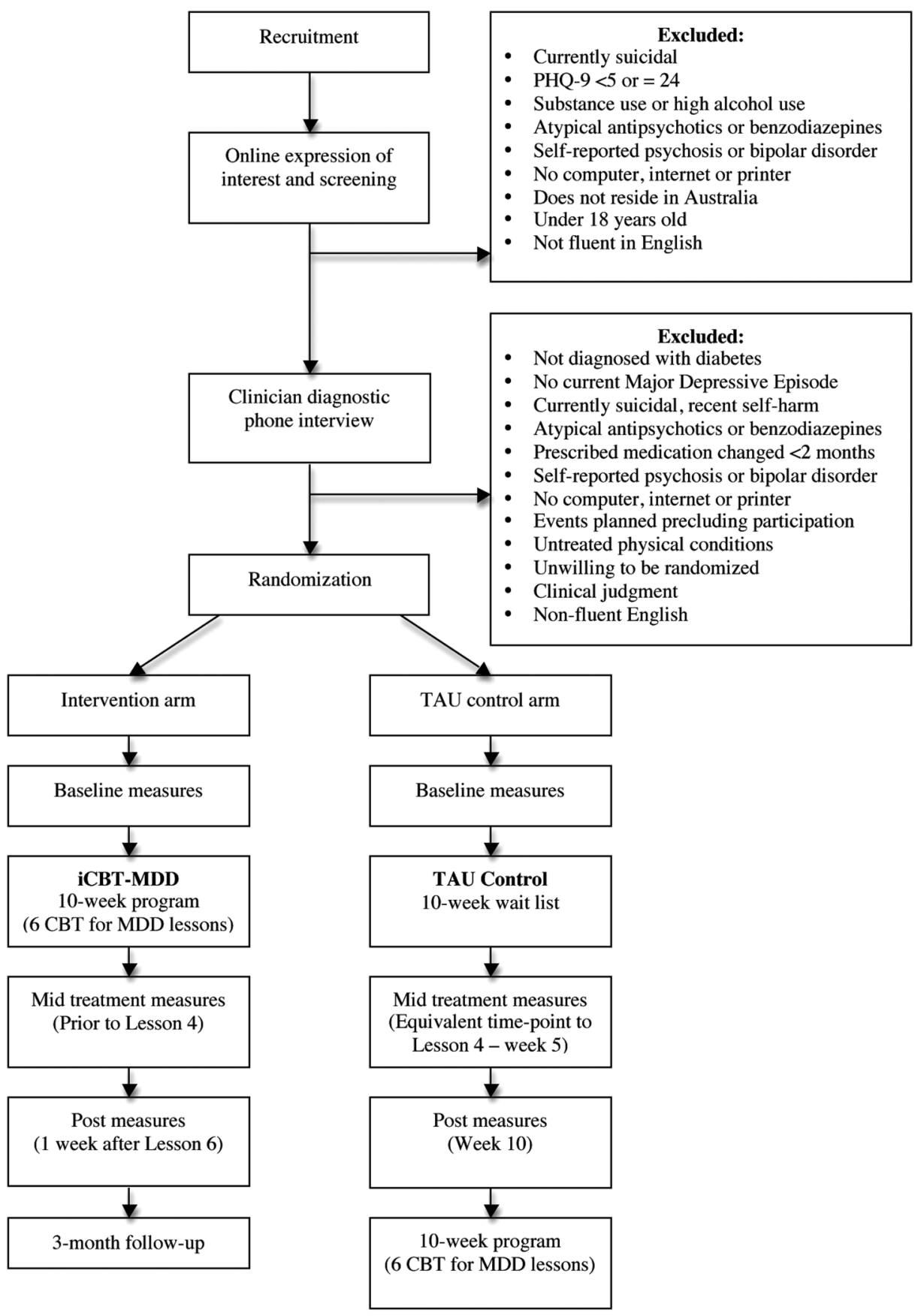


in response to participant request, or if initiated by the clinician. The TAU group will not receive any additional treatment during this period, but will be monitored pretrial, mid-trial and post-trial and offered further assistance if their condition deteriorates.

After the treatment group has finished the programme, the TAU group will be offered the iCBT-MDD programme. The treatment group will then complete the follow-up questionnaires and phone interview at 3 months post-treatment. See figure 1 for a procedural overview of the trial.

\section{Trial status}

The trial is currently in the data collection phase. Recruitment to the study started in February, 2014. A total of 334 people have volunteered to participate in the study and started an online application. Of those who have volunteered to take part, 168 have been screened, with 96 meeting inclusion criteria. It is anticipated that full post and follow-up data will be finalised in November, 2015.

\section{DISCUSSION}

This paper presents an overview of the iCBT-MDD programme and a description of the methods to implement and evaluate it in a population of adults living with comorbid depression and DM. The iCBT-MDD programme is standard of care treatment for depression in the Anxiety and Depression Clinic of St Vincent's Hospital Australia and is available to the Australian public under their clinician's supervision via ThisWayUp Clinic (https://thiswayupclinic.org). Because the iCBT-MDD programme is both affordable and easily accessible, it has the potential to serve as either an adjunct or stand-alone intervention for people with depression seeking treatment and/or looking for alternatives to traditional face-to-face offerings.

Evaluation of this programme in people with DM will provide evidence as to whether unmodified iCBT for depression is a suitable intervention, or if a more tailored CBT intervention specifically addressing the needs of this group is required. It is possible that the iCBT-MDD programme may require additional content that specifically addresses the relationship between depressive symptoms and struggling with a chronic illness and its management in order to improve self-care and DM outcomes. ${ }^{56}$

We hope to demonstrate the value of iCBT-MDD for use in primary care settings and DM services to assist general practitioners, DM educators, and endocrinologists to facilitate access to depression treatment. If evidence from this study suggests the programme is not suitable for people with DM, the reasons behind this will be explored to inform development of a more tailored CBT intervention for this group which includes illness-specific content.
Acknowledgements This research is being conducted by the authors as part of their employment with St Vincent's Hospital Australia. The authors would like to acknowledge the support of St Vincent's Hospital Australia. They acknowledge the contribution of Inika Gillis in conducting some of the clinician interviews. They also thank all the participants who took part in this research.

Contributors JN and GA conceived of the study. JN, KW, LR, and GA initiated the study design and JN, TF, JS, AF, TM, KW, and LR helped with implementation. JN provided statistical expertise in clinical trial design and JN and LR will conduct the primary statistical analysis. JN, LR, KW, and LC contributed to refinement of the study protocol and all authors approved the final manuscript.

Funding JN is supported by an Australian NHMRC Early Career Australian Clinical Fellowship (1037797). LR is supported by a grant from the St Vincent's Clinic Foundation.

\section{Competing interests None declared.}

Ethics approval Ethics approval has been obtained from the Human Research Ethics Committee of St Vincent's Hospital Australia (HREC/13/SVH/291). Results of this study will be disseminated via publication in peer-reviewed journals and conference presentations, and through the social media channels of St Vincent's Hospital Sydney and Consumer Representative Bodies for people with DM.

Provenance and peer review Not commissioned; externally peer reviewed.

Data sharing statement No additional data are available.

Open Access This is an Open Access article distributed in accordance with the Creative Commons Attribution Non Commercial (CC BY-NC 4.0) license, which permits others to distribute, remix, adapt, build upon this work noncommercially, and license their derivative works on different terms, provided the original work is properly cited and the use is non-commercial. See: http:// creativecommons.org/licenses/by-nc/4.0/

\section{REFERENCES}

1. International Diabetes Federation. IDF Diabetes Atlas. 6th edn. Brussels, Belgium: International Diabetes Federation, 2013.

2. Egede LE, Ellis C. Diabetes and depression: global perspectives. Diabetes Res Clin Pract 2010;87:302-12.

3. Moussavi S, Chatterji S, Verdes E, et al. Depression, chronic diseases, and decrements in health: results from the World Health Surveys. Lancet 2007;370:851-8.

4. Baumeister $\mathrm{H}$, Hutter $\mathrm{N}$, Bengel J, et al. Quality of life in medically ill persons with comorbid mental disorders: a systematic review and meta-analysis. Psychother Psychosom 2011;80:275-86.

5. Hermanns N, Kulzer B, Krichbaum M, et al. How to screen for depression and emotional problems in patients with diabetes: comparison of screening characteristics of depression questionnaires, measurement of diabetes-specific emotional problems and standard clinical assessment. Diabetologia 2006;49:469-77.

6. Gonzalez JS, Peyrot M, McCarl LA, et al. Depression and diabetes treatment nonadherence: a meta-analysis. Diabetes Care 2008;31:2398-403.

7. Davydow DS, Russo JE, Ludman E, et al. The association of comorbid depression with intensive care unit admission in patients with diabetes: a prospective cohort study. Psychosomatics 2011;52:117-26.

8. Hutter N, Schnurr A, Baumeister $\mathrm{H}$. Healthcare costs in patients with diabetes mellitus and comorbid mental disorders-a systematic review. Diabetologia 2010;53:2470-9.

9. Egede LE, Zheng D, Simpson K. Comorbid depression is associated with increased health care use and expenditures in individuals with diabetes. Diabetes Care 2002;25:464-70.

10. Lustman PJ, Anderson RJ, Freedland KE, et al. Depression and poor glycemic control: a meta-analytic review of the literature. Diabetes Care 2000;23:934-42.

11. Dirmaier J, Watzke B, Koch $\mathrm{U}$, et al. Diabetes in primary care: prospective associations between depression, nonadherence and glycemic control. Psychother Psychosom 2010;79:172-8.

12. Egede LE, Nietert PJ, Zheng D. Depression and all-cause and coronary heart disease mortality among adults with and without diabetes. Diabetes Care 2005;28:1339-45. 
13. Richardson LK, Egede LE, Mueller M. Effect of race/ethnicity and persistent recognition of depression on mortality in elderly men with type 2 diabetes and depression. Diabetes Care 2008;31:880-1.

14. Baumeister $\mathrm{H}$, Hutter N, Bengel J. Psychological and pharmacological interventions for depression in patients with diabetes mellitus and depression. Cochrane Database Syst Rev 2012;12:CD008381.

15. Van Der Feltz-Cornelis CM, Nuyen J, Stoop C, et al. Effect of interventions for major depressive disorder and significant depressive symptoms in patients with diabetes mellitus: a systematic review and meta-analysis. Gen Hosp Psychiatry 2010;32:380-95.

16. Speight J, Browne JL, Holmes-Truscott E, et al. Diabetes MILESAustralia 2011 survey report. Canberra: Diabetes Australia, 2011.

17. Poulsen KM, Pachana NA, McDermott BM. Health professionals' detection of depression and anxiety in their patients with diabetes the influence of patient, illness and psychological factors. J Health Psychol 2014:1-10.

18. Sorkin DH, Ngo-Metzger Q, Billimek J, et al. Underdiagnosed and undertreated depression among racially/ethnically diverse patients with type 2 diabetes. Diabetes Care 2011;34:598-600.

19. Nam S, Chesla C, Stotts NA, et al. Barriers to diabetes management: patient and provider factors. Diabetes Res Clin Pract 2011;93:1-9.

20. Parikh PB, Yang J, Leigh S, et al. The impact of financial barriers on access to care, quality of care and vascular morbidity among patients with diabetes and coronary heart disease. J Gen Intern Med 2014;29:76-81.

21. Petrak F, Baumeister H, Skinner TC, et al. Depression and diabetes: treatment and health-care delivery. Lancet Diabetes Endocrinol 2015;3:472-85

22. Andersson G, Cuijpers $P$, Carlbring $P$, et al. Guided internet-based vs face-to-face cognitive behavior therapy for psychiatric and somatic disorders: a systematic review and meta-analysis. World Psychiatry 2014:13:288-95.

23. Andrews $\mathrm{G}$, Titov $\mathrm{N}$. Is internet treatment for depressive and anxiety disorders ready for prime time? Med J Aust 2010;192(11 Suppl): S45-7.

24. Hedman E, Ljótsson B, Kaldo V, et al. Effectiveness of internet-based cognitive behaviour therapy for depression in routine psychiatric care. J Affect Disord 2014;155:49-58.

25. Titov N, Dear BF, Staples LG, et al. MindSpot clinic: an accessible, efficient, and effective online treatment service for anxiety and depression. Psychiatr Serv 2015;66:1043-50.

26. Watts S, Newby JM, Mewton L, et al. A clinical audit of changes in suicide ideas with internet treatment for depression. BMJ Open 2012;2: 001558.

27. Fisher L, Mullan JT, Arean P, et al. Diabetes distress but not clinical depression or depressive symptoms is associated with glycemic control in both cross-sectional and longitudinal analyses. Diabetes Care 2010;33:23-8.

28. Roy T, Lloyd CE. Epidemiology of depression and diabetes: a systematic review. J Affect Disord 2012;142:S8-21.

29. Fisher L, Skaff MM, Mullan JT, et al. Clinical depression versus distress among patients with type 2 diabetes: not just a question of semantics. Diabetes Care 2007;30:542-8.

30. Fisher L, Skaff MM, Mullan JT, et al. A longitudinal study of affective and anxiety disorders, depressive affect and diabetes distress in adults with type 2 diabetes. Diabet Med 2008;25:1096-101.

31. van Bastelaar KM, Pouwer F, Geelhoed-Duijvestijn PH, et al. Diabetes-specific emotional distress mediates the association between depressive symptoms and glycaemic control in type 1 and type 2 diabetes. Diabet Med 2010;27:798-803.

32. Schmitt A, Reimer A, Kulzer B, et al. Negative association between depression and diabetes control only when accompanied by diabetes-specific distress. J Behav Med 2015;38:556-64.

33. Snoek FJ, Bremmer MA, Hermanns N. Constructs of depression and distress in diabetes: time for an appraisal. Lancet Diabetes Endocrinol 2015;3:450-60.

34. van Bastelaar KM, Pouwer F, Cuijpers $P$, et al. Web-based depression treatment for type 1 and type 2 diabetic patients: a randomized, controlled trial. Diabetes Care 2011;34:320-5
35. van Bastelaar KM, Pouwer F, Cuijpers $P$, et al. Is a severe clinical profile an effect modifier in a web-based depression treatment for adults with type 1 or type 2 diabetes? Secondary analyses from a randomized controlled trial. J Med Internet Res 2012;14:e2.

36. Nobis S, Lehr D, Ebert DD, et al. Efficacy of a web-based intervention with mobile phone support in treating depressive symptoms in adults with type 1 and type 2 diabetes: a randomized controlled trial. Diabetes Care 2015;38:776-83.

37. Chan A-W, Tetzlaff JM, Altman DG, et al. SPIRIT 2013 Statement: defining standard protocol items for clinical trials. Ann Intern Med 2013;158:200-7.

38. Perini S, Titov N, Andrews G. Clinician-assisted internet-based treatment is effective for depression: a randomized controlled trial. Aust N Z J Psychiatry 2009;43:571-8.

39. Moher D, Hopewell S, Schulz KF, et al. CONSORT 2010 explanation and elaboration: updated guidelines for reporting paralle group randomised trials. BMJ 2010;340:c869.

40. Sheehan DV, Lecrubier Y, Sheehan $\mathrm{KH}$, et al. The mini-international neuropsychiatric interview (m.I.N.I): The development and validation of a structured diagnostic psychiatric interview for dsm-iv and icd-10. J Clin Psychiatry 1998;59(Suppl 20):22-33.

41. Richards D, Richardson T. Computer-based psychological treatments for depression: a systematic review and meta-analysis. Clin Psychol Rev 2012;32:329-42.

42. Clarke G, Eubanks D, Reid E, et al. Overcoming depression on the internet (ODIN) (2): a randomized trial of a self-help depression skills program with reminders. J Med Internet Res 2005;7:e16.

43. Titov N, Dear BF, Johnston L, et al. Improving adherence and clinical outcomes in self-guided internet treatment for anxiety and depression: randomised controlled trial. PLOS ONE 2013;8:e62873.

44. Williams AD, Andrews $\mathrm{G}$. The effectiveness of internet cognitive behavioural therapy (iCBT) for depression in primary care: a quality assurance study. PLOS ONE 2013;8:e57447.

45. Kroenke K, Spitzer R, Williams J. The PHQ-9: validity of a brief depression severity measure. J Gen Intern Med 2001;16:606-13.

46. Sheehan DV, Lecrubier $\mathrm{Y}$, Sheehan $\mathrm{KH}$, et al. The Mini-International Neuropsychiatric Interview (MINI): the development and validation of a structured diagnostic psychiatric interview for DSM-IV and ICD-10. J Clin Psychiatry 1998;59:22-33.

47. Kessler RC, Ustun TB. The world mental health (WMH) survey initiative version of the world health organization (WHO) composite international diagnostic interview (CIDI). Int J Methods Psychiatr Res 2004;13:93-121.

48. Welch GW, Jacobson AM, Polonsky WH. The problem areas in diabetes scale. An evaluation of its clinical utility. Diabetes Care 1997;20:760-6.

49. Welch GW, Weinger BA, Polonsky WH. Responsiveness of the problem areas in diabetes (PAID) questionnaire. Diabet Med 2003;20:69-72.

50. Kessler R, Andrews G, Colpe L, et al. Short screening scales to monitor population prevalences and trends in non-specific psychological distress. Psychol Med 2002;32:959-76.

51. Wilson DM, Ciliska D. Lifestyle assessment: development and use of the fantastic checklist. Can Fam Physician 1984;30:1527-32.

52. Ware JE, Kosinski M, Keller S. A 12-Item Short-Form Health Survey: construction of scales and preliminary tests of reliability and validity. Med Care 1996;34:220-33.

53. Spitzer RL, Williams JB, Kroenke $\mathrm{K}$, et al. Validity and utility of the prime-md patient health questionnaire in assessment of 3000 obstetric-gynecologic patients: the prime-md patient health questionnaire obstetrics-gynecology study. Am J Obstet Gynecol 2000;183:759-69.

54. Spitzer RL, Kroenke K, Williams JB, et al. A brief measure for assessing generalized anxiety disorder: the gad-7. Arch Intern Med 2006;166:1092-7.

55. Lyons-Ruth K, Holmes BM. The Relationship QuestionnaireClinical Version (RQ-CV). Introducing a profoundly distrustful attachment style. Infant Ment Health J 2006;27:310-25.

56. Gonzalez JS, Fisher L, Polonsky WH. Depression in diabetes: have we been missing something important? Diabetes Care 2011;34:236-9. 